\title{
A Nash Equilibrium Analysis for Interference Coupled Wireless Systems
}

\author{
Siddharth Naik \\ Technical University of Berlin \\ Heinrich Hertz Chair for Mobile Communications \\ Einsteinufer 25, \\ 10587 Berlin, Germany \\ naik@hhi.fraunhofer.de
}

\author{
Tansu Alpcan \\ Technical University of Berlin \\ Deutsche Telekom Laboratories \\ Ernst-Reuter Platz 7, \\ 10587 Berlin, Germany \\ alpcan@sec.t-labs.tu-berlin.de
}

\author{
Holger Boche \\ Technical University of Berlin \\ Heinrich Hertz Institute \\ Einsteinufer 25, \\ 10587 Berlin, Germany \\ holger.boche@mk.tu-berlin.de
}

\begin{abstract}
This paper studies the properties of Nash equilibrium for noncooperative games in interference coupled wireless systems, where it serves as an incentive-compatible solution concept and an operating point. A broad class of noncooperative power control games played among the users of the wireless system is defined based on a general interference function framework, which models the interference coupling through a set of axioms. Both cases of coupling with and without selfinterference are considered. The special properties of the underlying interference functions as well as relevant sufficient conditions are investigated to establish the existence and uniqueness of a Nash equilibrium solution. These properties play an important role in developing incentive-compatible distributed algorithms for a variety of wireless networks with interference coupling.
\end{abstract}

\section{INTRODUCTION}

Decentralized and user-centric wireless systems will be more the norm than exception in the future. Such systems naturally require decentralized algorithms for achieving the desired outcome of resource allocation strategies. The participating users are typically selfish, i.e. utility maximizing, and have limited information about each other. Simultaneously, such systems are typically coupled with interference. Hence, noncooperative game theory has been utilized in the analysis of such interference-limited wireless systems. The concept of Nash equilibrium is relevant and useful in describing the incentive-compatible operating points of such games. Furthermore, the existence and uniqueness properties of Nash equilibrium play an important role in developing incentivecompatible distributed algorithms for a variety of wireless networks with interference coupling.

\section{A. Background}

There exists a large body of literature on noncooperative game theoretic issues within the context of wireless communication systems, [1]. Most of this work deals with networking aspects of communications. However, there is also certain work done in relation to the interference frequency channels (IFC). Our reference list is by no means comprehensive and is only a brief survey of the work done in the domain of IFC.

Siddharth Naik was supported by the DFG project B0 1734/17-1.

Holger Boche and Tansu Alpcan were partly supported by the Deutsche Telekom Laboratories.
Distributed algorithms for spectrum sharing in a competitive setup (using noncooperative game theory [2]) were developed by [3] and [4]. A more general analysis of the spectrum sharing problem was performed in [5]. All three publications [3][5] dealt with single-antenna transmitters and receivers, and looked at the problem from a noncooperative game-theoretic point of view. The multiple-input-multiple-output (MIMO) IFC has also been studied from a noncooperative gametheoretic perspective in [6] and [7], which presented results on equilibrium rates and proposed distributed algorithms. The noncooperative approaches of [3]-[6], [8] generally lead to decentralized schemes for computing stable operating points, so-called Nash equilibria. The study [9] shows how centralized and decentralized power control algorithms in wireless communications can be viewed as S-modular games coupled policy sets. The papers [10]-[16] have considered various problems in wireless communications such as uplink power control and pricing issues in a noncooperative setting.

This paper provides a characterization of the conditions on interference coupled wireless systems, with and without self interference, which result in a unique Nash equilibrium for a broad class of noncooperative power control games. Such a framework can be potentially applied to various power control games, where the assumptions specified in this paper are satisfied. We have utilized an interference function framework to model interference coupling in wireless systems. This interference function framework has been introduced and well motivated previously in literature for various problems in wireless systems. The standard interference functions have been introduced in [17] and extended in [18].

\section{B. Contributions}

The main contributions of this paper are as follows:

- Investigation of certain basic properties of interference functions relevant to the game theoretic analysis.

- Study of general conditions sufficient for the existence of a unique Nash equilibrium for convex, compact strategy sets in interference coupled wireless systems.

- Exploration of the properties of interference functions, that need to be satisfied for a unique Nash equilibrium to exist in interference coupled wireless systems. 
The rest of the paper is organized as follows:

- Section II describes certain notation and preliminaries used in our paper. It introduces the interference function framework and the axioms, which are used to model interference coupling in our paper.

- Section III investigates certain basic properties of interference functions and signal to interference ratio functions derived from interference functions. It provides certain practical examples of the consequences of the results.

- Section IV provides the noncooperative game theoretic framework, which we utilize to investigate the existence and uniqueness of Nash equilibrium in interference coupled wireless systems. We provide examples of systems with individual and total power constraints for obtaining our convex and compact strategy sets.

- Section V displays the conditions required on the interference functions to obtain a negative definite matrix and utilizes previous results to show existence and uniqueness of the Nash equilibrium in interference coupled wireless systems.

\section{INTERFERENCE FUNCTION FRAMEWORK}

The notation used in this paper is summarized as follows: The mapping $\mathcal{I}$ represents interference functions. The scalar $K$ is the number of users in the system. Let $\boldsymbol{y}$ be a vector, then $y_{k}=[\boldsymbol{y}]_{k}$ is the $k^{\text {th }}$ component. Likewise $G_{j k}=[\boldsymbol{G}]_{j k}$ is the $j k^{\text {th }}$ component of the matrix $\boldsymbol{G}$. The notation $\boldsymbol{y} \geq \mathbf{0}$ implies that $y_{k} \geq 0$ for all components $k . \boldsymbol{x} \geq \boldsymbol{y}$ implies component-wise inequality with strict inequality for at least one componets. Similar definitions hold for the reverse directions. $\boldsymbol{x} \neq \boldsymbol{y}$ implies that the vectors differ in at least one component. The set of non-negative reals is denoted as $\mathbb{R}_{+}$. The set of positive reals is denoted as $\mathbb{R}_{++}$.

The signal-to-interference (plus noise) ratio (SINR) is an important measure for user performance in wireless systems. Many other performance measures have a direct relationship with SINR. Consider $K$ users with transmit powers $\boldsymbol{p}=$ $\left[p_{1}, \ldots, p_{K}\right]^{T}$ and $\mathcal{K}:=\{1, \ldots, K\}$. The noise power at each receiver is $\sigma^{2}$. Hence, the SINR at each receiver depends on the extended power vector $\boldsymbol{p}=\left[p_{1}, \ldots, p_{K}, \sigma^{2}\right]^{T}$. The resulting SINR of user $k$ is

$$
\operatorname{SINR}_{k}(\underline{\boldsymbol{p}})=\frac{p_{k}}{\mathcal{I}_{k}(\underline{\boldsymbol{p}})}
$$

where $\mathcal{I}_{k}(\boldsymbol{p})$ is the interference (plus noise) as a function of $\boldsymbol{p}$. Let $\operatorname{SINR}_{k}(\underline{\boldsymbol{p}}):=s_{k}(\underline{\boldsymbol{p}})$ for notational convenience. In order to model interference, we shall follow the axiomatic approach proposed in [17], [18]. Let $\mathcal{P}$ be the set of all power vectors. In our paper, we have $\mathcal{P}:=\mathbb{R}_{+}^{K+1}$ unless explicitly mentioned otherwise.

Definition 1 . We say that $\mathcal{I}: \mathcal{P} \mapsto \mathbb{R}_{+}$is an interference function, if the following axioms are fulfilled:

$$
\begin{array}{cl}
A 1 & \text { conditional positivity } \mathcal{I}(\underline{\boldsymbol{p}})>0 \text { if } \underline{\boldsymbol{p}}>\mathbf{0} \\
A 2 & \text { scale invariance } \mathcal{I}(\alpha \underline{\boldsymbol{p}})=\alpha \mathcal{I}(\underline{\boldsymbol{p}}), \forall \alpha \in \mathbb{R}_{+} \\
A 3 & \text { monotonicity } \mathcal{I}(\underline{\boldsymbol{p}}) \geq \mathcal{I}(\underline{\hat{\boldsymbol{p}}}) \text { if } \underline{\boldsymbol{p}} \geq \underline{\hat{\boldsymbol{p}}} \\
A 4 & \text { strict monotonicity } \mathcal{I}(\underline{\boldsymbol{p}})>\mathcal{I}(\underline{\hat{\boldsymbol{p}}}) \text { if } \underline{\boldsymbol{p}} \geq \underline{\hat{\boldsymbol{p}}}, \\
& \underline{\underline{p}}_{K+1}>\underline{\hat{p}}_{K+1} .
\end{array}
$$

Note that we require that $\mathcal{I}(\boldsymbol{p})$ is strict monotone with respect to the last component $\underline{p}_{K+1}$. An example is $\mathcal{I}(\underline{\boldsymbol{p}})=$ $\boldsymbol{v}^{T} \underline{\boldsymbol{p}}$ where $\boldsymbol{v} \in \mathbb{R}_{+}^{K+1}$ is a vector of interference coupling coefficients. The axiomatic framework A1-A4 is connected with the framework of standard interference functions [17].

Definition 2. A function $Y: \mathcal{P} \mapsto \mathbb{R}_{++}$, where $\mathcal{P}=\mathbb{R}_{+}^{K}$, is said to be a standard interference function if the following axioms are fulfilled:

$$
\begin{array}{ll}
Y 1 & \text { positivity } Y(\boldsymbol{p})>0, \text { for all } \boldsymbol{p} \in \mathbb{R}_{+}^{K}, \\
Y 2 & \text { scalability } Y(\alpha \boldsymbol{p})<\alpha Y(\boldsymbol{p}), \text { for all } \alpha>1, \\
Y 3 & \text { monotonicity } Y(\boldsymbol{p}) \geq Y(\hat{\boldsymbol{p}}) \text { if } \boldsymbol{p} \geq \hat{\boldsymbol{p}} .
\end{array}
$$

Any standard interference function can be expressed within the general interference function framework. The details about the relationship between the model A1-A3 and Yates' standard interference functions are further investigated in [19]. We note that the results of this paper are also applicable to standard interference functions. We begin our investigation by presenting an analysis of general SINR functions in Section III below.

\section{AnAlysis of General SINR Functions}

In this section we shall analyze certain basic properties of general SINR functions, with the help of the properties of the underlying general interference functions. We assume that the function $\mathcal{I}_{k}(\underline{p})$ is smooth and multiple times differentiable, i.e. $\mathcal{I}_{k} \in C^{2}$. Then, taking the partial derivative of $s_{k}$ with respect to power $p_{k}$ of the $k^{\text {th }}$ user, we have that

$$
\frac{\partial s_{k}}{\partial p_{k}}=\frac{\mathcal{I}_{k}(\underline{\boldsymbol{p}})-p_{k} \frac{\partial \mathcal{I}_{k}}{\partial p_{k}}}{\mathcal{I}_{k}^{2}(\underline{\boldsymbol{p}})} .
$$

We now state a simple lemma in relation to the effect of the power $p_{k}$ of the $k^{\text {th }}$ user on the SINR function $s_{k}$ of the $k^{\text {th }}$ user.

Lemma 1. The function $s_{k}$ given by (1) is increasing in $p_{k}$, if and only if $\mathcal{I}_{k}(\underline{\boldsymbol{p}})>p_{k} \partial \mathcal{I}_{k} / \partial p_{k}$. Similarly, $s_{k}$ is nondecreasing in $p_{k}$, if and only if $\mathcal{I}_{k}(\underline{p}) \geq p_{k} \partial \mathcal{I}_{k} / \partial p_{k}$.

Proof: The proof follows immediately from the derivative.

Note that, if $\partial \mathcal{I}_{k} / \partial p_{k}=0$, we have the case with interference functions without any self-interference. In this case, it is obvious that $s_{k}$ is an increasing function of $p_{k}$.

Example 1. Consider the case of linear interference functions $\mathcal{I}_{k}(\underline{\boldsymbol{p}})=\sum_{k \in \mathcal{K}}\left|h_{j}\right|^{2} p_{j}$. Hence from Lemma 1 we have the condition that $\sum_{j \in \mathcal{K}}\left|h_{j}\right|^{2} p_{j}-p_{k}\left|h_{k}\right|^{2}>0$. 
Now we take the second derivative of (2) with respect to the power $p_{k}$ of the $k^{\text {th }}$ user. Then, we have that

$$
\frac{\partial^{2} s_{k}}{\partial p_{k}^{2}}=\frac{\mathcal{I}_{k}(\underline{\boldsymbol{p}})\left(-p_{k} \frac{\partial^{2} \mathcal{I}_{k}(\underline{\boldsymbol{p}})}{\partial p_{k}^{2}}-2 \frac{\partial \mathcal{I}_{k}(\underline{\boldsymbol{p}})}{\partial p_{k}}\right)+2 p_{k}\left(\frac{\partial \mathcal{I}_{k}(\underline{\boldsymbol{p}})}{\partial p_{k}}\right)^{2}}{\mathcal{I}_{k}^{3}(\underline{\boldsymbol{p}})} \text {. }
$$

Furthermore, to investigate the effect of the other users on $s_{k}$ we take the derivative of (1) with respect to the power $p_{j}$ of the $j^{\text {th }}$ user, where $j \in \mathcal{K}$ and $j \neq k$. Then, we have that

$$
\frac{\partial s_{k}}{\partial p_{j}}=\frac{-p_{k} \frac{\partial \mathcal{I}_{k}}{\partial p_{j}}}{\mathcal{I}_{k}^{2}(\underline{\boldsymbol{p}})} .
$$

Lemma 2. The function $s_{k}$ is increasing in $p_{j}, j \neq k$, if and only if $\partial \mathcal{I}_{k} / \partial p_{j}<0$. Similarly, the function $s_{k}$ is nondecreasing in $p_{j}$, if and only if $\partial \mathcal{I}_{k} / \partial p_{j} \leq 0$.

The function $s_{k}$ is decreasing in $p_{j}$, if and only if $\partial \mathcal{I}_{k} / \partial p_{j}>0$. Similarly, the function $s_{k}$ is non-increasing in $p_{j}$, if and only if $\partial \mathcal{I}_{k} / \partial p_{j} \geq 0$.

We skip the proof, which is a direct consequence of basic calculus, due to space limitations.

Remark 1. From the axiom $A 3$ of interference functions, we can never have that $\partial \mathcal{I}_{k} / \partial p_{j}<0$ or that $\partial \mathcal{I}_{k} / \partial p_{j} \leq 0$. Hence, the function $s_{k}$ is a decreasing or non-increasing function of power $p_{j}$ of the $j^{\text {th }}$ user.

Remark 2. For the case of no self-interference, it is trivial that $s_{k}$ is a decreasing function of $p_{j}$.

Theorem 1 . If the interference function $\mathcal{I}_{k}$ is a (strictly) convex interference function and

$$
p_{k} \mathcal{I}_{k}(\underline{\boldsymbol{p}}) \frac{\partial^{2} \mathcal{I}_{k}(\underline{\boldsymbol{p}})}{\partial p_{k}^{2}}+2 \frac{\partial \mathcal{I}_{k}(\underline{\boldsymbol{p}})}{\partial p_{k}}>2 p_{k}\left(\frac{\partial \mathcal{I}_{k}(\underline{\boldsymbol{p}})}{\partial p_{k}}\right)^{2}
$$

then the function $s_{k}$ is strictly (concave) with respect to $p_{k}$.

Proof: After suitable re-arrangement of terms in (3) we have that $\partial^{2} \mathcal{I}_{k}$ is positive if $\mathcal{I}_{k}$ is strictly convex. Then, we only require the condition in the statement of the Theorem.

Example 2. For the case of linear interference functions $\mathcal{I}_{k}=\sum_{j \in \mathcal{K}}\left|h_{j}\right|^{2} p_{j}$, we have from Theorem 1 that the power allocation should follow the rule:

$$
p_{k}<\frac{1}{\left|h_{k}\right|^{2}}, \quad \forall k \in \mathcal{K} .
$$

We now take the derivative of (2) with respect to $p_{j}$. Then, we have that

$$
\frac{\partial^{2} s_{k}}{\partial p_{k} \partial p_{j}}=\frac{2 p_{k} \frac{\partial \mathcal{I}_{k}}{\partial p_{k}} \frac{\partial \mathcal{I}_{k}}{\partial p_{j}}-\mathcal{I}_{k}(\underline{\boldsymbol{p}}) \frac{\partial \mathcal{I}_{k}}{\partial p_{k}}-p_{k} \mathcal{I}_{k}(\underline{\boldsymbol{p}}) \frac{\partial^{2} \mathcal{I}_{k}}{\partial p_{k} \partial p_{j}}}{\mathcal{I}_{k}^{3}(\underline{\boldsymbol{p}})} .
$$

Theorem 2. The function $\partial^{2} s_{k} / \partial p_{k} \partial p_{j}$ in (5)

- takes a zero value, if there is no self-interference,

- takes a negative value, if there is self-interference and $\mathcal{I}_{k}(\underline{\boldsymbol{p}})>2 p_{k} \frac{\partial \mathcal{I}_{k}}{\partial p_{k}}$,

- takes a positive value, if there is self-interference and $\mathcal{I}_{k}(\underline{\boldsymbol{p}})<2 p_{k} \frac{\partial \mathcal{I}_{k}}{\partial p_{k}}$

Proof: The three statements of the theorem can be easily verified by substituting the conditions in (5).

\section{Game Theoretic Model and Nash Equilibrium}

In this section we investigate the existence and uniqueness of a Nash equilibrium solution to a generic noncooperative game in an interference coupled wireless system. We define a broad class of "utility" functions for users of the game that depend on their individual SINR by a strictly monotone and continuous function $\phi$, which is defined on $\mathbb{R}_{+}$. The utility of user $k$ is

$$
u_{k}(\underline{\boldsymbol{p}})=\phi_{k}\left(s_{k}(\underline{\boldsymbol{p}})\right), \quad k \in \mathcal{K} .
$$

An example of the above case if capacity: $\phi(x)=\log (1+$ $x)$. In the following performance indicators, we would like to minimize the objective function, e.g. MMSE: $\phi(x)=1 /(1+$ $x)$, BER: $\phi(x)=Q(\sqrt{x})$ and high-SNR approximation of BER $\phi(x)=x^{-\alpha}$ with diversity order $\alpha$.

We consider the following game, where at each time instant the users channel gains are fixed, and individual users adjust only their powers in their corresponding strategy spaces $\mathbf{P}_{k}$ (power domain), in order to maximize their corresponding utility functions.

The strategy space for the case of individual power constraints, which have been normalized is given as follows:

$$
\mathbf{P}_{k}=\left\{p_{k} \in \mathbb{R}_{+} \mid p_{k} \in\left(0, \hat{p}_{k}\right]\right\}, \quad \forall k \in \mathcal{K},
$$

where $\hat{p}_{k}$ is the individual maximum power for the $k^{\text {th }}$ user.

Similarly, we can write the strategy sets in the power domain for the following cases:

- total power constraint $P_{\text {total }}$ on the system;

$$
\mathbf{P}=\left\{\boldsymbol{p} \in \mathbb{R}_{+}^{K} \mid\|p\|_{1} \leq P_{\text {total }}\right\},
$$

where $P_{\text {total }}$ is the total power constraint and $\mathbf{P}:=$ $\times_{k \in \mathcal{K}} \mathbf{P}_{k}$.

- total power constraint $P_{\text {total }}$ on the system and individual power constraint on each user.

The noncooperative game, $\mathcal{G}$, in the power domain is formally defined as follows.

$$
\mathcal{G}\left(\mathcal{K},\left\{u_{k}(\underline{\boldsymbol{p}})\right\}_{k \in \mathcal{K}}\right):\left\{\mathbf{P}_{k}\right\}_{k \in \mathcal{K}} \rightarrow\left\{\mathbf{P}_{k}\right\}_{k \in \mathcal{K}}
$$

where the three components of the noncooperative game are as follows:

1) $\mathcal{K}$ is the set of active users at each time instant,

2) $\mathbf{P}_{k}$ is the set of strategies in the power domain for each user $k$ and

3) $u_{k}(\underline{\boldsymbol{p}})$ is the user utility function that maps the joint strategy space into the set of non-negative real numbers

Individual users select their strategies to maximize their corresponding cost functions for a given set of powers, i.e.

$$
\max _{p_{k} \in \mathbf{P}_{k}} u_{k}(\underline{\boldsymbol{p}}), \quad \forall k \in \mathcal{K} .
$$

In order to investigate the existence of Nash equilibrium for the noncooperative game defined in (9) we state the following formal definitions from noncooperative game theory in the context of our problem. 
Definition 3. Nash equilibrium for the power game : A power vector $p^{*}$ is a Nash equilibrium of the power game, if for every user $k \in \mathcal{K}$ we have that

$$
\begin{aligned}
& u_{k}\left(p_{1}^{*}, \ldots, p_{k-1}^{*}, p_{k}^{*}, p_{k+1}^{*}, \ldots, p_{K}^{*}, \sigma^{2}\right) \geq \\
& \quad u_{k}\left(p_{1}^{*}, \ldots, p_{k-1}^{*}, p_{k}, p_{k+1}^{*}, \ldots, p_{K}^{*}, \sigma^{2}\right), \forall p_{k} \in \mathbf{P} .
\end{aligned}
$$

The Nash equilibrium of the noncooperative game $\mathcal{P}$ is defined as the strategy vector, $\boldsymbol{p}^{*}$. Furthermore, we have the corresponding set of costs $\boldsymbol{u}^{*}$, with the property that no user can benefit from modifying its strategy, while the other users keep their strategies fixed.

We make the following assumptions on the utility functions of the users and the strategy space of the users

- The strategy space $\mathbf{P}$ of the power game is convex, compact and has a non-empty interior $\operatorname{int}(\mathbf{P}) \neq \emptyset$.

- The utility function $u_{k}$ of the $k^{\text {th }}$ user is twice continuously differentiable in all its arguments.

We now take the derivative of the utility function $\phi_{k}$ with respect to $p_{k}$. Then, we have that

$$
\begin{aligned}
\frac{\partial \phi_{k}}{\partial p_{k}} & =\phi^{\prime} \frac{\partial s_{k}}{\partial p_{k}} \\
\frac{\partial^{2} \phi_{k}}{\partial p_{k}^{2}} & =\phi^{\prime \prime}\left(\frac{\partial s_{k}}{\partial p_{k}}\right)^{2}+\phi^{\prime} \frac{\partial^{2} s_{k}}{\partial p_{k}^{2}} \\
\frac{\partial^{2} \phi_{k}}{\partial p_{k} \partial p_{j}} & =\phi^{\prime \prime}\left(\frac{\partial s_{k}}{\partial p_{k}} \frac{\partial s_{k}}{\partial p_{j}}\right)+\phi^{\prime} \frac{\partial^{2} s_{k}}{\partial p_{k} \partial p_{j}},
\end{aligned}
$$

where $\phi^{\prime}:=\partial \phi_{k} / \partial s_{k}$ and $\phi^{\prime \prime}:=\partial^{2} \phi_{k} / \partial s_{k}^{2}$. We now define the pseudo-gradient operator $\bar{\nabla}$ through its application on the utility $\boldsymbol{u}$ as follows:

$$
\bar{\nabla} \boldsymbol{u}:=\left[\partial u_{1}(\underline{\boldsymbol{p}}) / \partial p_{1}, \ldots, \partial u_{K}(\underline{\boldsymbol{p}}) / \partial p_{K}\right]^{T}:=\boldsymbol{g}(\underline{\boldsymbol{p}}) .
$$

Let $\boldsymbol{G}(\boldsymbol{p})$ be the pseudo-Jacobian of $\boldsymbol{g}(\boldsymbol{p})$ with respect to the power vector $\boldsymbol{p}$. Hence, we have that

$$
\boldsymbol{G}(\underline{\boldsymbol{p}})=\left(\begin{array}{cccc}
b_{1} & a_{12} & \ldots & a_{1 K} \\
\vdots & b_{2} & \ddots & \vdots \\
a_{K 1} & a_{K 2} & \ldots & b_{K}
\end{array}\right),
$$

where $b_{k}:=\partial^{2} \phi_{k}(\underline{\boldsymbol{p}}) / \partial p_{k}^{2}$ and $a_{k j}:=\partial^{2} \phi_{k}(\underline{\boldsymbol{p}}) / \partial p_{k} \partial p_{j}$ respectively. Based on (13), we define the symmetric matrix

$$
\boldsymbol{J}(\underline{\boldsymbol{p}})=\boldsymbol{G}(\underline{\boldsymbol{p}})+\boldsymbol{G}(\underline{\boldsymbol{p}})^{T} .
$$

We briefly contrast the scenario with [20]. The paper [20] investigates the possibility of having joint concavity or joint convexity of functions of SINR and functions of inverse SINR, respectively. In our paper we investigate properties with respect to a user's own parameter. The difference in the two approaches is due to the fact, that here we are looking to find a Nash equilibrium to a non-cooperative game, which might not be efficient (on the boundary of the strategy set). While [20] looks to find problems, where one can ensure that a local optimum is the global optimum. Hence, we utilize the pseudoJacobian instead of the Jacobian.
Lemma 3. Let the functions $\phi_{k}$ and $s_{k}$ be strictly concave and non-decreasing in their arguments $s_{k}$ and $p_{k}$ respectively. Then, in (13) $b_{k}<0$ for all $k \in \mathcal{K}$.

Theorem 3. Let the domain of the game be compact and the utility function $u_{k}$ for all $k \in \mathcal{K}$ be concave in the power $p_{k}$ of the $k^{\text {th }}$ user. Then, the game admits a Nash equilibrium solution.

Proof: The proof is provided in [21].

The proofs of the following theorems can be found in [22].

Theorem 4. Let the matrix $G$ in (13) be full rank. Then, there can be at most a single inner Nash equilibrium.

Corollary 1. Let the matrix $G$ defined in (14) be either positive definite or negative definite. Then, there can be at most a single inner Nash equilibrium.

\section{Negative Definite MatriX - Interference FUNCTION FRAMEWORK}

Based on the insight and results presented in the previous section, we are now in a position to present our main results in relation to existence and uniqueness of a Nash equilibrium in certain interference coupled wireless systems. Before we delve into our analysis, we briefly recap the definitions of a negative definite matrix and diagonally dominant matrix, which we shall utilize, while obtaining our results.

Definition 4. Negative definite matrix: An $K \times K$ Hermitian matrix $G$ is said to be negative definite if

$$
\boldsymbol{x}^{T} \boldsymbol{G} \boldsymbol{x}<0,
$$

for all non-zero vectors $\boldsymbol{x} \in \mathbb{R}^{K}$.

If the strict inequality required in (15) is weakened to $\boldsymbol{x}^{T} \boldsymbol{G} \boldsymbol{x} \leq 0$, then $\boldsymbol{G}$ is said to be negative semi-definite. Of course, if $G$ is negative definite, then it is also negative semidefinite.

Definition 5. Diagonally dominant matrix: Let $\boldsymbol{G}=[\boldsymbol{G}]_{k j} \in$ $M_{K}$. The matrix $G$ is said to be diagonally dominant if

$$
\left|[\boldsymbol{G}]_{k k}\right| \geq \sum_{j \in \mathcal{K} \backslash k}\left|[\boldsymbol{G}]_{k j}\right|,
$$

for all $k \in \mathcal{K}$.

We attempt to find the conditions on our matrix $G$ in (13) such that it is a negative semi-definite or negative definite matrix. We shall see, in the following result that, the general case is quite involved. Hence, we restrict ourselves to analyze the case, when all the users have the same utility function, i.e. $\phi_{1}=\ldots=\phi_{K}$.

Theorem 5. Let all the users have the same utility function, i.e. $\phi_{1}=\ldots=\phi_{K}$. Then, if

$$
\mathcal{I}_{k}(\underline{\boldsymbol{p}}) \geq \sum_{j \in \mathcal{K}} p_{k} \frac{\partial \mathcal{I}_{k}}{\partial p_{j}}
$$


and

$$
\begin{aligned}
& \left|2 p_{k}\left(\frac{\partial \mathcal{I}_{k}}{\partial p_{k}}\right)^{2}-p_{k} \mathcal{I}_{k}(\underline{\boldsymbol{p}}) \frac{\partial^{2} \mathcal{I}_{k}}{\partial p_{k}^{2}}-2 \mathcal{I}_{k}(\underline{\boldsymbol{p}}) \frac{\partial \mathcal{I}_{k}}{\partial p_{k}}\right| \geq \\
& \sum_{j \in \mathcal{K} \backslash k}\left|2 p_{k} \frac{\partial \mathcal{I}_{k}}{\partial p_{k}} \frac{\partial \mathcal{I}_{k}}{\partial p_{J}}-p_{k} \mathcal{I}_{k}(\underline{\boldsymbol{p}}) \frac{\partial^{2} \mathcal{I}_{k}}{\partial p_{k} \partial p_{j}}-\mathcal{I}_{k}(\underline{\boldsymbol{p}}) \frac{\partial \mathcal{I}_{k}}{\partial p_{j}}\right|
\end{aligned}
$$

are fulfilled - the noncooperative power control game defined in (9) has a unique Nash equilibrium.

Proof: We have that, the utility functions of all the users be the same, i.e. $\phi_{1}=\ldots=\phi_{K}$. Then, we have that the matrix $G$ is a symmetric matrix. We now analyze the following expressions:

$$
\begin{aligned}
b_{k} & =\phi^{\prime \prime}\left(\frac{\partial s_{k}}{\partial p_{k}}\right)^{2}+\phi^{\prime} \frac{\partial^{2} s_{k}}{\partial p_{k}^{2}} \\
a_{k j} & =\phi^{\prime \prime}\left(\frac{\partial s_{k}}{\partial p_{k}} \frac{\partial s_{k}}{\partial p_{j}}\right)+\phi^{\prime} \frac{\partial^{2} s_{k}}{\partial p_{k} \partial p_{j}} .
\end{aligned}
$$

In order $G$ to satisfy the condition of diagonal dominance, we have to compare $\left|b_{k}\right|$ and $\sum_{j \in \mathcal{K} \backslash k}\left|a_{k j}\right|$. Therefore, it is sufficient to have the following two inequalities:

$$
\begin{aligned}
& \left(\frac{\partial s_{k}}{\partial p_{k}}\right)^{2} \geq \sum_{j \in \mathcal{K} \backslash k}\left|\frac{\partial s_{k}}{\partial p_{k}} \frac{\partial s_{k}}{\partial p_{j}}\right| \\
& \left|\frac{\partial^{2} s_{k}}{\partial p_{k}^{2}}\right| \geq \sum_{j \in \mathcal{K} \backslash k}\left|\frac{\partial^{2} s_{k}}{\partial p_{k} \partial p_{j}}\right| .
\end{aligned}
$$

For (18) to hold we must have that

$$
\begin{aligned}
& \left|\frac{\mathcal{I}_{k}(\underline{\boldsymbol{p}})-p_{k} \frac{\partial \mathcal{I}_{k}}{\partial p_{k}}}{\mathcal{I}_{k}^{2}(\underline{\boldsymbol{p}})}\right| \geq\left|\frac{-p_{k} \frac{\partial \mathcal{I}_{k}}{\partial p_{j}}}{\mathcal{I}_{k}^{2}(\underline{\boldsymbol{p}})}\right| \\
& \left|\mathcal{I}_{k}(\underline{\boldsymbol{p}})-p_{k} \frac{\partial \mathcal{I}_{k}}{\partial p_{k}}\right| \geq\left|-p_{k} \frac{\partial \mathcal{I}_{k}}{\partial p_{j}}\right| .
\end{aligned}
$$

We know that

$$
\left|\mathcal{I}_{k}(\underline{\boldsymbol{p}})-p_{k} \frac{\partial \mathcal{I}_{k}}{\partial p_{k}}\right| \geq\left|\mathcal{I}_{k}(\underline{\boldsymbol{p}})\right|-\left|p_{k} \frac{\partial \mathcal{I}_{k}}{\partial p_{k}}\right| .
$$

Therefore, from (20) and (21), the condition required for (18) to hold is that:

$$
\mathcal{I}_{k}(\underline{\boldsymbol{p}}) \geq \sum_{j \in \mathcal{K}} p_{k} \frac{\partial \mathcal{I}_{k}}{\partial p_{j}}
$$

Similarly, it can be shown that, for the condition (19) to be fulfilled, the following condition has to be satisfied:

$$
\begin{aligned}
& \left|2 p_{k}\left(\frac{\partial \mathcal{I}_{k}}{\partial p_{k}}\right)^{2}-p_{k} \mathcal{I}_{k}(\underline{\boldsymbol{p}}) \frac{\partial^{2} \mathcal{I}_{k}}{\partial p_{k}^{2}}-2 \mathcal{I}_{k}(\underline{\boldsymbol{p}}) \frac{\partial \mathcal{I}_{k}}{\partial p_{k}}\right| \geq \\
& \sum_{j \in \mathcal{K} \backslash k}\left|2 p_{k} \frac{\partial \mathcal{I}_{k}}{\partial p_{k}} \frac{\partial \mathcal{I}_{k}}{\partial p_{J}}-p_{k} \mathcal{I}_{k}(\underline{\boldsymbol{p}}) \frac{\partial^{2} \mathcal{I}_{k}}{\partial p_{k} \partial p_{j}}-\mathcal{I}_{k}(\underline{\boldsymbol{p}}) \frac{\partial \mathcal{I}_{k}}{\partial p_{j}}\right|
\end{aligned}
$$

From, [23] page 349, Theorem 6.1.10.c, we have the following result. Let $\boldsymbol{G}=[\boldsymbol{G}]_{j k} \in \mathbb{R}^{K}$ be (strictly) diagonally dominant. Then, if the matrix $G$ is Hermetian and all the main diagonal entries of $\boldsymbol{G}$ are negative, then all the eigenvalues of $\boldsymbol{G}$ are real and (negative) non-positive.
Let $\boldsymbol{G}=[\boldsymbol{G}]_{j k} \in \mathbb{R}^{K}$ be (strictly) diagonally dominant, symmetric and all the main diagonal entries of $G$ are negative. Hence, the matrix $G$ is negative (definite) semi-definite, which completes the proof.

Example 3. Consider for the case of two users, the utility function $u_{1}\left(p_{1}, p_{2}\right)=\log \left(\left|h_{1}\right|^{2} p_{1} /\left|h_{2}\right|^{2} p_{2}\right)$. Then, we have that

$$
\begin{aligned}
\frac{\partial u_{1}}{\partial p_{1}} & =\frac{1}{p_{1}} \\
\frac{\partial u_{2}}{\partial p_{2}} & =\frac{1}{p_{2}} \\
\frac{\partial^{2} u_{1}}{\partial p_{1}^{2}} & =-\frac{1}{p_{1}^{2}} \\
\frac{\partial^{2} u_{2}}{\partial p_{2}^{2}} & =-\frac{1}{p_{2}^{2}} .
\end{aligned}
$$

Then, we have that the matrix $\boldsymbol{G}$ can be written as follows:

$$
\boldsymbol{G}=\left[\begin{array}{cc}
-\frac{1}{p_{1}^{2}} & 0 \\
0 & -\frac{1}{p_{2}^{2}}
\end{array}\right]
$$

which is negative semi-definite.

Example 4. Consider the following utility function for the case of two users.

$$
u_{1}=\log \left(\frac{\left|h_{1}\right|^{2} p_{1}}{\left|h_{1}\right|^{2} p_{1}+\left|h_{2}\right|^{2} p_{2}}\right) .
$$

We have a similar a utility function for user 2 . Then, we have that

$$
\begin{aligned}
\frac{\partial u_{1}}{\partial p_{1}} & =\frac{\left|h_{2}\right|^{2} p_{2}}{p_{1}\left(\left|h_{1}\right|^{2} p_{1}+\left|h_{2}\right|^{2} p_{2}\right)} \\
\frac{\partial u_{2}}{\partial p_{2}} & =\frac{\left|h_{1}\right|^{2} p_{1}}{p_{2}\left(\left|h_{1}\right|^{2} p_{1}+\left|h_{2}\right|^{2} p_{2}\right)} \\
\frac{\partial^{2} u_{1}}{\partial p_{1}^{2}} & =\frac{-2\left|h_{1}\right|^{2}\left|h_{2}\right|^{2}}{p_{1}\left(\left|h_{1}\right|^{2} p_{1}+\left|h_{2}\right|^{2} p_{2}\right)} \\
\frac{\partial^{2} u_{2}}{\partial p_{2}^{2}} & =\frac{-2\left|h_{1}\right|^{2}\left|h_{2}\right|^{2}}{p_{2}\left(\left|h_{1}\right|^{2} p_{1}+\left|h_{2}\right|^{2} p_{2}\right)} \\
\frac{\partial^{2} u_{1}}{\partial p_{1} p_{2}} & =\frac{\left|h_{1}\right|^{2}\left|h_{2}\right|^{2}}{\left|h_{1}\right|^{2} p_{1}+\left|h_{2}\right|^{2} p_{2}} \\
\frac{\partial^{2} u_{2}}{\partial p_{2} p_{1}} & =\frac{\left|h_{1}\right|^{2}\left|h_{2}\right|^{2}}{\left|h_{1}\right|^{2} p_{1}+\left|h_{2}\right|^{2} p_{2}} .
\end{aligned}
$$

Then, we have that the matrix $\boldsymbol{G}$ can be written as follows:

$$
\boldsymbol{G}=\left[\begin{array}{cc}
\frac{-2\left|h_{1}\right|^{2}\left|h_{2}\right|^{2}}{p_{1}\left(\left|h_{1}\right|^{2} p_{1}+\left|h_{2}\right|^{2} p_{2}\right)} & \frac{\left|h_{1}\right|^{2}\left|h_{2}\right|^{2}}{\left|h_{1}\right|^{2} p_{1}+\left|h_{2}\right|^{2} p_{2}} \\
\frac{\left|h_{1}\right|^{2}\left|h_{2}\right|^{2}}{\left|h_{1}\right|^{2} p_{1}+\left|h_{2}\right|^{2} p_{2}} & \frac{-2\left|h_{1}\right|^{2}\left|h_{2}\right|^{2}}{p_{2}\left(\left|h_{1}\right|^{2} p_{1}+\left|h_{2}\right|^{2} p_{2}\right)}
\end{array}\right] .
$$

It can be shown that, the matrix $\boldsymbol{G}$ is negative (semi)-definite, if and only if $p_{1}, p_{2} \leq 2$. Furthermore, if we analyze the determinant of $\boldsymbol{G}$ for negative (semi)-definiteness, we obtain the following condition $p_{1} p_{2} \leq 4$, which is a less restrictive condition than the previous one.

Hence, it can be observed from the above examples, that self interference can lead to complications and unforeseen constraints in interference coupled wireless systems. 


\section{Discussion}

In this paper we have studiedcertain basic properties of the interference functions, which provide an axiomatic framework for modeling interference coupling in wireless systems. A class of noncooperative power control games has been defined. The basic properties of interference functions along with certain other conditions have been utilized to prove certain desirable properties of the pseudo-Jacobian matrix of the noncooperative power control game. The properties of existence and uniqueness of the Nash equilibrium play an important role in developing incentive-compatible distributed algorithms for a variety of wireless networks with interference coupling. The obtained results have been elucidated with certain examples of utility functions which also highlight the effect of the presence of self interference in the system.

\section{REFERENCES}

[1] A. MacKenzie and L. DaSilva, Game Theory for Wireless Engineers. Morgan and Claypool Publishers, 2006.

[2] T. Basar and G. J. Olsder, Dynamic Noncooperative Game Theory, 2nd ed. SIAM.

[3] W. Yu, W. Rhee, S. Boyd, and J. Cioffi, "Iterative water-filling for Gaussian vector multiple access channels," IEEE Transactions on Information Theory, vol. 50, pp. 145-151, January 2004.

[4] J. Huang, R. A. Berrz, and M. L. Honig, "Distributed interference compensation for wireless networks," IEEE Journal on Selected Areas in Communications, vol. 24, pp. 1074-1084, May 2006.

[5] R. Etkin, A. Parekh, and D. Tse, "Spectrum sharing for unlicensed bands," IEEE Journal on Selected Areas in Communications, vol. 25, pp. $517-528,2007$.

[6] G. Arslan, M. F. Demirkol, and Y. Song, "Equilibrium efficiency improvement in mimo interference systems: a decentralized stream control approach," IEEE Trans. on Wireless Communications, 2006.

[7] D. P. P. G. Scutari and S. Barbarossa, "Optimal linear precoding strategies for wideband non-cooperative systems based on game-theory - part I: Nash Equilibria," IEEE Transactions on Signal Processing, 2007.

[8] F. Meshkati, M. Chiang, S. C. Schwartz, H. V. Poor, and N. B. Mandayam, "A non-cooperative power control game for multi-carrier cdma systems," arXiv:cs/0501064v1.

[9] E. Altman and Z. Altman, "S-modular games and power control in wireless networks," IEEE Transactions on Automatic Control, vol. 48, no. 5, May 2003.

[10] C. U. Saraydar, N. B. Mandayam, and D. J. Goodman, "Power Control via Pricing in Wireless Data Networks," IEEE Transactions on Communications, vol. 50, no. 2, pp. 291-303, February 2002.

[11] _ "Pricing and Power Control in Multicell Wireless Data Network," IEEE Journal on Selected Areas in Communications, vol. 19, no. 10, pp. 1883-1892, October 2001.

[12] M. Cesana, N. Gatti, and I. Malanchini, "A Game-Theoretic Approach to Decentralized Optimal Power Allocation for Cellular Networks," in International Workshop on Game Theory in Networks, Athens, Greece, October 2008.

[13] T. Alpcan, T. Basar, and S. Dey, "A power control game based on outage probabilities for multicell wireless data networks," IEEE Transactions on Wireless Communications, vol. 5, no. 4, pp. 890-899, April 2006.

[14] T. Alpcan, X. Fan, T. Başar, M. Arcak, and J. T. Wen, "Power control for multicell CDMA wireless networks: A team optimization approach," Wireless Networks, vol. 14, no. 5, pp. 647-657, October 2008.

[15] T. Alpcan and T. Başar, "A hybrid systems model for power control in multicell wireless data networks," Performance Evaluation, vol. 57, no. 4, pp. 477-495, August 2004.

[16] T. Alpcan, T. Başar, R. Srikant, and E. Altman, "CDMA uplink power control as a noncooperative game," Wireless Networks, vol. 8, no. 6, pp. 659-670, November 2002.

[17] R. D. Yates, "A framework for uplink power control in cellular radio systems," IEEE Journal on Selected Areas in Communication, vol. 13, no. 7, pp. 1341-1348, September 1995.
[18] M. Schubert and H. Boche, "QoS-based resource allocation and transceiver optimization." Foundations and Trends in Communications and Information Theory, 2005/2006, vol. 2, no. 6, pp. 383-529.

[19] H. Boche and M. Schubert, "Unified Framework for Interference Modeling of Multi-User Wireless Networks," in IEEE ICC, Dresden, Germany, June 2009.

[20] H. Boche and S. Naik, "Impact of Interference Coupling - Loss of Convexity," in IEEE Globecom, Honolulu, Hawaii, USA, December 2009.

[21] T. Alpcan, Noncooperative Games for Control of Networked Systems, Urbana, IL, May 2006.

[22] T. Alpcan and T. Basar, Annals of the International Society of Dynamic Games, Advances in Dynamic Games. Birkhaueser Boston, 2005. vol. 7, ch. Distributed Algorithms for Nash Equilibrium for Flow Control Games, pp. 473-498.

[23] R. Horn and C. Johnson, Matrix Analysis. Cambridge University Press, 1987. 